\title{
Electronic coherence transfer in photosynthetic complexes and its signatures in optical spectroscopy
}

\author{
Tomáš Mančal $^{\text {a,* }}$, Leonas Valkunas ${ }^{\text {b,c }}$, Elizabeth L. Read ${ }^{\text {d,e }}$, Gregory S. Engel ${ }^{\text {d,e }}$, \\ Tessa R. Calhoun ${ }^{\mathrm{d}, \mathrm{e}}$ and Graham R. Fleming d,e \\ ${ }^{a}$ Institute of Physics, Faculty of Mathematics and Physics, Charles University in Prague, Prague, \\ Czech Republic \\ ${ }^{\mathrm{b}}$ Institute of Physics, Vilnius, Lithuania \\ ${ }^{\mathrm{c}}$ Department of Theoretical Physics, Faculty of Physics of Vilnius University, Vilnius, Lithuania \\ ${ }^{\mathrm{d}}$ Department of Chemistry, University of California, Berkeley, CA, USA \\ ${ }^{\mathrm{e}}$ Physical Biosciences Division, Lawrence Berkeley National Laboratory, Berkeley, CA, USA
}

\begin{abstract}
Effects of electronic coherence transfer after photoexcitation of excitonic complexes and their manifestation in optical spectroscopy are discussed. A general excitonic model Hamiltonian is considered in detail to elucidate the origin of energy relaxation in excitonic complexes. We suggest that the second-order quantum master equation for the reduced density matrix of electronic degrees of freedom provides the most suitable theoretical framework for the study of coherence transfer in photosynthetic bacteriochlorophyll complexes. Temperature dependence of the absorption band maximum of a simple excitonic dimer is interpreted in terms of coherence transfer between two excited states. The role of reorganization energy of the transitions in the magnitude of the effect is discussed. A large reorganization energy difference between the two states is found to induce significant band shift. The predictions of the theory are compared to experimental measurements of the bacterial reaction center absorption spectra of Rhodobacter sphaeroides. As an example of a time-dependent spectroscopic method sensitive to coherences and possibly to their transfer, we present recent two-dimensional photon echo measurements of energy relaxation in the so-called Fenna-Matthews-Olson complex of Chlorobium tepidum, where distinct oscillatory patters predicted to be signatures of electronic coherence have been observed.
\end{abstract}

Keywords: Electronic coherence, coherence transfer, relaxation dynamics, photosynthetic complexes, optical spectroscopy

\section{Introduction}

Photosynthetic organisms exhibit a variety of mechanisms to convert the energy of light into its chemical form suitable for running vital cellular processes [1]. In chlorophyll based photosynthesis, light harvesting antenna complexes collect the sunlight and deliver excitation energy to reaction centers in a highly effective way [2]. Observation of such processes in an ensemble of photosynthetic systems and in ambient light results necessarily in observation of averaged out dynamics that seems to be fully described by the population kinetics of electronically excited states. The source of the averaging is twofold.

\footnotetext{
${ }^{*}$ Corresponding author: Tomáš Mančal, Institute of Physics, Faculty of Mathematics and Physics, Charles University in Prague, Ke Karlovu 5, CZ-121 16 Prague, Czech Republic. Tel.: +420 221911 337; Fax: +420 224922 797; E-mail: mancal@karlov.mff.cuni.cz.
} 
First, the ensemble of molecules is characterized by disorder (e.g. in excitation energies) due to varying environment, and second, the sun as a thermal source of light is exciting the members of a molecular ensemble at random times. A short laser pulse sets the same origin of time (within the pulse duration) for the evolution of each molecule in the sample. Thus, an ideal ensemble of molecules (with no additional disorder) would act in coherence like a single molecule. Due to the presence of disorder, however, this ideal case is almost never realized in natural systems, and very fast decay of coherence, usually termed dephasing, is observed. Some techniques of ultrafast spectroscopy provide means for eliminating the contribution of certain sources of disorder. Most notably, photon echo and its variety of applications is capable of removing the energetic disorder, so-called inhomogeneous spectral broadening. Recently, techniques of two-dimensional heterodyne detected Fourier transformed spectroscopy have been realized in the optical and near infrared spectral regions [3-6] and applied to study of photosynthetic complexes [7-10]. The great sensitivity of the two-dimensional photon echo to coherence is underlined by its ability to detect the presence of electronic wavepackets in excited states of excitonically coupled molecular systems [11]. The observation of such coherent wavepackets in the photosynthetic bacteriochlorophyll (BChl)-protein complex FMO [8] suggested the importance of coherence in the energy transfer efficiency of photosynthesis. Recently, coherent dynamics has been observed also in bacterial reaction centers (BRC) by means of two-color photon echo peakshift measurements [12].

Another type of effect that is connected to the notion of coherence has been observed in the absorption spectrum of BRC. In [13,14], a temperature dependent shift of the lowest energy band of the BRC was shown to be a result of interaction of excitonic levels with a charge transfer (CT) state. The effect could be attributed to coherence transfer between the excitonic and CT transitions [14]. As temperature dependencies of absorption spectra are fairly common among photosynthetic systems [15-17] the development of a general theoretical framework that can deal with such effects is of eminent importance. Although most of the measurements of coherent effects are performed outside the physiological range of temperatures, they provide valuable insight into the inner workings of photosynthesis on the level of individual molecules. Time-dependent measurements like those of $[8,12]$ are especially suggestive that coherence may play a significant role in achieving photosynthesis' remarkable energy transfer efficiency. In this paper, our primary goal is to provide the simplest possible framework for understanding coherence transfer effects.

\section{Relaxation of energy and coherence transfer in excitonic systems}

\subsection{Origin of energy relaxation in excitonic systems}

In excitonic systems, electronic transitions of molecules lose their individuality in a complex. Due to the mutual electrostatic interaction of the molecules, delocalized electronic eigenstates (excitons) are formed in the complex [2]. Dynamics of excited state population in natural excitonic systems is characterized by very fast, even sub picosecond, energy relaxation. This energy relaxation is a consequence of electronic ground-to excited state transition energy fluctuations of individual molecules, which in turn is due to interaction of their electronic degrees of freedom (DOF) with internal nuclear DOF and the DOF of their immediate surroundings.

Let us assume an aggregate of $N$ molecules embedded in an environment, such as a protein matrix or solvent. In order to study energy relaxation among its electronic states, we split the total Hamiltonian of the system into a purely electronic part $H_{\mathrm{S}}$, a part that contains pure contribution of all other DOF, 
$H_{\mathrm{B}}$, and an interaction part $H_{\mathrm{S}-\mathrm{B}}$ that specifies the coupling between the two pure parts of the molecular system. Further on in this paper, all the DOF included in $H_{\mathrm{B}}$ will be referred to as the bath or environment of the relevant or system DOF included in $H_{\mathrm{S}}$. For the total Hamiltonian we can formally write $H=$ $H_{\mathrm{S}}+H_{\mathrm{B}}+H_{\mathrm{S}-\mathrm{B}}$. To provide a specific form of $H_{\mathrm{S}}, H_{\mathrm{B}}$ and $H_{\mathrm{S}-\mathrm{B}}$ we first perform similar formal splitting of the Hamiltonian on a single molecule denoted by index $\alpha$ (a monomer), while neglecting its interaction with the other $N-1$ molecules of the aggregate. The Hamiltonian of the monomer can be written as $H_{\alpha}\left(P_{\alpha}, Q_{\alpha}\right)=T\left(P_{\alpha}\right)+\left[\epsilon_{\alpha}^{g}+V_{\alpha}^{g}\left(Q_{\alpha}\right)\right]\left|g_{\alpha}\right\rangle\left\langle g_{\alpha}\left|+\left[\epsilon_{\alpha}^{e}+V_{\alpha}^{e}\left(Q_{\alpha}\right)\right]\right| e_{\alpha}\right\rangle\left\langle e_{\alpha}\right|$, where $P_{\alpha}$ and $Q_{\alpha}$ are impulses and coordinates of the nuclei that form the bath for the electronic states $\left|g_{\alpha}\right\rangle$ and $\left|e_{\alpha}\right\rangle$ with respective electronic energies $\epsilon_{\alpha}^{g}$ and $\epsilon_{\alpha}^{e}$, and potential energy surfaces $V_{\alpha}^{g}\left(Q_{\alpha}\right)$ and $V_{\alpha}^{e}\left(Q_{\alpha}\right)$ (see e.g. $[2,18]$ ). $T$ denotes kinetic energy of the nuclei. This Hamiltonian can be formally split into the required pure electronic, pure bath and interaction terms. We define the so-called energy gap operator $\Delta V\left(Q_{\alpha}\right)=V_{\alpha}^{e}\left(Q_{\alpha}\right)-V_{\alpha}^{g}\left(Q_{\alpha}\right)-\left\langle V_{\alpha}^{e}\left(Q_{\alpha}\right)-V_{\alpha}^{g}\left(Q_{\alpha}\right)\right\rangle_{Q_{\alpha}}$, where the brackets $\langle\cdots\rangle_{Q_{\alpha}}$ denote averaging over all nuclear DOF $Q_{\alpha}$ in equilibrium. The total Hamiltonian is then a sum of Hamiltonian operators:

$$
\begin{aligned}
& H_{\mathrm{B}}^{\alpha}=T\left(Q_{\alpha}\right)+V_{\alpha}^{g}\left(Q_{\alpha}\right), \\
& H_{\mathrm{S}}^{\alpha}=\epsilon_{\alpha}^{g}\left|g_{\alpha}\right\rangle\left\langle g_{\alpha}\left|+\left[\epsilon_{\alpha}^{e}+\left\langle V_{\alpha}^{e}\left(Q_{\alpha}\right)-V_{\alpha}^{g}\left(Q_{\alpha}\right)\right\rangle_{Q_{\alpha}}\right]\right| e_{\alpha}\right\rangle\left\langle e_{\alpha}\right|, \\
& H_{\mathrm{S}-\mathrm{B}}^{\alpha}=\Delta V_{\alpha}\left(Q_{\alpha}\right)\left|e_{\alpha}\right\rangle\left\langle e_{\alpha}\right| .
\end{aligned}
$$

The system-bath interaction Hamiltonian $H_{\mathrm{S}-\mathrm{B}}^{\alpha}$ contains only the excited state projector and cannot produce transitions between the states $\left|g_{\alpha}\right\rangle$ and $\left|e_{\alpha}\right\rangle$. If the difference of the ground state and the excited state potentials is small, the interaction term is small and perturbation theory can be used to derive the effect of the interaction term on the system evolution in the excited state.

Building an aggregate from the two-level molecules results in a mutual electrostatic interaction among the monomers described by some Hamiltonian $H_{\text {int }}^{\text {agg }}$ containing resonance coupling terms [2]. This interaction leads to energy level splitting and formation of delocalized eigenstates of the system. The Hilbert space of states of such an aggregate consists of all possible states of the monomers. These include the aggregate ground state, where no monomer is excited, and the aggregate excited states, where one or more excited monomers are present. Using the basis states with up to two excitations

$$
|\overline{0}\rangle=\prod_{\alpha=1}^{N}\left|g_{\alpha}\right\rangle, \quad|\bar{\alpha}\rangle=\left|e_{\alpha}\right\rangle \prod_{\beta \neq \alpha}^{N}\left|g_{\beta}\right\rangle, \quad|(\alpha, \beta)\rangle=\left|e_{\alpha}\right\rangle\left|e_{\beta}\right\rangle \prod_{\gamma \neq \alpha, \beta}^{N}\left|g_{\gamma}\right\rangle
$$

we arrive at an electronic Hamiltonian of the aggregate

$$
H_{\mathrm{S}}^{\mathrm{agg}}=\sum_{\alpha} \epsilon_{e}^{\alpha}|\bar{\alpha}\rangle\left\langle\bar{\alpha}\left|+\sum_{\alpha<\beta}\left[\epsilon_{e}^{\alpha}+\epsilon_{e}^{\beta}\right]\right|(\alpha, \beta)\right\rangle\langle(\alpha, \beta)|+H_{\mathrm{int}}^{\mathrm{agg}}
$$

and the aggregate-bath interaction Hamiltonian in the form

$$
H_{\mathrm{S}-\mathrm{B}}^{\mathrm{agg}}=\sum_{\alpha=1}^{N} \Delta V_{\alpha}\left(Q_{\alpha}\right)|\bar{\alpha}\rangle\left\langle\bar{\alpha}\left|+\sum_{\alpha<\beta}\left[\Delta V_{\alpha}\left(Q_{\alpha}\right)+\Delta V_{\beta}\left(Q_{\beta}\right)\right]\right|(\alpha, \beta)\right\rangle\langle(\alpha, \beta)| .
$$

The states $|\overline{0}\rangle,|\overline{1}\rangle, \ldots,|\bar{N}\rangle$ and the corresponding two excitation states are not the electronic eigenstates of the system due to the presence of the coupling term $H_{\mathrm{int}}^{\mathrm{agg}}$ in the Hamiltonian, Eq. (5). In general, 
this Hamiltonian term contains off-diagonal electrostatic, such as dipole-dipole, coupling terms. We switch into more convenient and more spectroscopically meaningful electronic eigenstate picture by diagonalizing the Hamiltonian of Eq. (5). By this procedure we arrive at new electronic states, so-called one-exciton and two-exciton states $|n\rangle$ and $|M\rangle$, respectively. Although the electronic Hamiltonian is now diagonal in the new basis, the system-bath interaction Hamiltonian has in general off-diagonal terms that will necessarily lead to transitions between electronic eigenstates. For the subsequent discussion of the system-bath coupling, we introduce operators

$$
K_{\alpha}=\sum_{n m} c_{\alpha}^{n}\left(c_{\alpha}^{m}\right)^{*}|n\rangle\left\langle m\left|, \quad K_{(\alpha, \beta)}=\sum_{M L} z_{\alpha \beta}^{M}\left(z_{\alpha \beta}^{L}\right)^{*}\right| M\right\rangle\langle L|,
$$

where the coefficients $c_{\alpha}^{n}$ and $z_{\alpha \beta}^{M}$ are expansion coefficient of the original electronic states in the new basis. The system-bath interaction Hamiltonian can be written as

$$
H_{\mathrm{S}-\mathrm{B}}^{\mathrm{agg}}=\sum_{k} \Delta V_{k}\left(Q_{k}\right) K_{k}
$$

where $k$ runs over all indices $\alpha=1, \ldots, N$ and $(\alpha, \beta)=(1,2),(1,3), \ldots,(N-1, N)$. For the energy gap operator $\Delta V_{(\alpha, \beta)}$ we write $\Delta V_{(\alpha, \beta)}=\Delta V_{\alpha}+\Delta V_{\beta}$. The above form of the aggregate-bath interaction Hamiltonian is a starting point for the development of systematic theories of the energy relaxation in the excitonic systems.

\subsection{Second-order system-bath interaction theories}

When the resonance coupling of the monomers in the aggregate is small in comparison with the system-bath coupling terms, the original localized electronic states can be to a good approximation considered electronic eigenstates of the system. The system-bath Hamiltonian then remains diagonal and we develop perturbation theory with respect to the resonance coupling Hamiltonian $H_{\text {int }}^{\text {agg }}$. Such a treatment leads to the Förster type of theories resulting in a prescription for population relaxation rates $[19,20]$. In the relaxation rate theories, no prescription is given for the relaxation of coherences, or their transfer between different sites and it is usually assumed that the coherences decay according to the standard rule, $\gamma_{\text {deph }} \equiv 1 / T_{\text {deph }}=(1 / 2) T_{\text {relax }}+1 / T^{*}$, where $T_{\text {relax }}$ is the population relaxation time and $T^{*}$ is some pure dephasing time.

In the opposite limit, when the resonance coupling is greater than that with the DOF of the environment, the excitonic basis provides the electronic eigenstates that are perturbed by the $H_{\mathrm{S}-\mathrm{B}}^{\mathrm{agg}}$ Hamiltonian. A rigorous theory can be developed using so-called projection operator techniques (see e.g. [2,21,22]). The most commonly used theory by far derived from this approach is the second order quantum master equation (QME) (see e.g. [21]) and the so-called Redfield equations, that can be obtained from the QME in Markov approximation. Here, population relaxation rates, dephasing rates and coherence transfer rates are all calculated in a unified manner, thus enabling the study of coherence transfer phenomena. To overcome the limitation of the second order theory with respect to the bath, one can either go into higher orders of the perturbation theory [23] or include partially the $H_{\mathrm{S}-\mathrm{B}}^{\mathrm{agg}}$ into the exact treatment. This was done by Zhang et al. [24] using a projector operator that projects on diagonal elements of the density matrix only, leading thus to a population rate theory. The population rates are calculated beyond second order in the system-bath coupling and it was shown [25] that they converge to both Förster (strong bath limit) and Redfield rates (weak bath limit) in their respective limits of validity. However, as this theory 
does not give any predictions for the dephasing and the coherence transfer rates it cannot be used in our discussion.

Another approach to the theory of relaxation dynamics that can in principle provide a basis for investigation of coherence transfer effects is the so-called Lindblad theory [21,26]. In this method, the relaxation operator is derived from the conditions on the positivity of the probabilities of the density matrix. In this theory we are again provided with all necessary rates, for all processes related to transfer and dephasing of coherences and at the same time the theory is not bound to any perturbative limit, however, since the down-hill and up-hill population transition rates resulting from this theory are the same the theory can only be valid in high temperature limit.

Thus, it seems that in the quest for understanding the effects of coherence transfer, the second-order QME and its higher order generalizations provide one of the few viable paths. Particularly, in the second order Markovian formulation of the theory, leading to time-independent Redfield tensor, we obtain theory simple enough to allow simple interpretation of observed effects, and in some case even derivation of analytical results.

\subsection{Quantum master equation and time-dependent Redfield tensor}

If a molecular system is described by the density matrix $W(t)$, evolution of the relevant system can be described by the reduced density matrix (RDM) of the electronic DOF $\rho(t)=\operatorname{Tr}_{Q}\{W\}$. The equation of motion for $\rho(t)$ can be derived by standard methods using projection operator technique and an expansion into second order in the system-bath coupling $H_{\text {int }}^{\text {agg }}$ (see e.g. [21]). The resulting integro-differential equation can be simplified by Markov approximation into

$$
\left.\frac{\partial}{\partial t} \rho_{a b}(t)\right|_{\text {diss }}=-\sum_{c d} R_{a b c d}(t) \rho_{c d}(t)
$$

where $R_{a b c d}(t)=\delta_{a c} \sum_{e} \Gamma_{b e e d}(t)+\delta_{b d} \sum_{e} \Gamma_{a e e c}(t)-\Gamma_{c a, d b}(t)-\Gamma_{d b, a c}(t)$ is the so-called time-dependent Redfield tensor. It is composed of complex quantities

$$
\Gamma_{a b c d}(t)=\sum_{n, m}\left\langle a\left|K_{m}\right| b\right\rangle\left\langle c\left|K_{n}\right| d\right\rangle \int_{0}^{t-t_{0}} \mathrm{~d} \tau C_{m n}(\tau) \mathrm{e}^{-\mathrm{i} \omega_{c d} \tau}
$$

where $C_{m n}(t)=\left\langle U_{\mathrm{B}}^{\dagger}(t) \Delta V_{m}\left(Q_{m}\right) U_{\mathrm{B}}(t) \Delta V_{n}\left(Q_{n}\right)\right\rangle$ are transition energy correlation functions and $U_{\mathrm{B}}(t)$ is the bath evolution operator. The correlation function $C_{m n}(t)$ determines the line shape of the transition in absence of relaxation via so-called line broadening function defined as $g_{n n}(t)=$ $\frac{1}{\hbar} \int_{0}^{t} \mathrm{~d} \tau \int_{0}^{\tau} \mathrm{d} \tau^{\prime} C_{n n}\left(\tau^{\prime}\right)$ (see e.g. [18]).

Using the above relaxation tensor, the evolution of an optical coherence element of the RDM can be written as

$$
\left.\frac{\partial}{\partial t} \rho_{a g}(t)\right|_{\text {diss }}=-\dot{g}_{a a}(t) \rho_{a g}(t)-\gamma_{a}(t) \rho_{a g}(t)-\sum_{c \neq a} \gamma_{a c}(t) \rho_{c g}(t)
$$


where, in the pure dephasing term (the first term of Eq. (11)), we have identified, $\Gamma_{a a a a}(t)$, with the first derivative of the line broadening function, $g_{a a}(t)$, of the excitonic state $|a\rangle$, and we defined

$$
\gamma_{a}(t)=\sum_{d \neq a} \Gamma_{a d d a}(t), \quad \gamma_{a c}(t)=\sum_{d} \Gamma_{a d d c}(t)
$$

Interpretation of $\gamma_{a}(t)$ will become clear from the following equation for the population evolution, and the quantity $\gamma_{a c}(t)$ can be readily interpreted as a coherence transfer rate between optical coherences, $\rho_{c g}(t)$ and $\rho_{a g}(t)$. The evolution of a population can be expressed as

$$
\begin{aligned}
\left.\frac{\partial}{\partial t} \rho_{a a}(t)\right|_{\text {diss }}= & -K_{a a}(t) \rho_{a a}(t)+\sum_{c \neq a} K_{a c}(t) \rho_{c c}(t)-\sum_{c \neq a}\left\{\gamma_{a c}(t) \rho_{c a}(t)+c . c .\right\} \\
& +\sum_{c d, c \neq a, b, d}\left[\Gamma_{a c a d}^{*}(t) \rho_{c d}(t)+c . c .\right]
\end{aligned}
$$

where $K_{a c}(t)$ is a time-dependent population relaxation rate between states $|a\rangle$ and $|c\rangle$. It can be shown that

$$
K_{a a}(t)=\sum_{b \neq a} K_{a c}=2 \operatorname{Re} \gamma_{a}(t)
$$

and $\gamma_{a}(t)$ of Eq. (12) can be interpreted as coherence decay rate due to population relaxation. We can also see that the population dynamics is influenced by coherence transfer processes. On the second line of Eq. (13) there is a contribution of conversion of a coherence $\rho_{a c}(t)$ into a population $\rho_{a a}(t)$ with the rate of a $\rho_{c g}(t)$ to $\rho_{a g}(t)$ process. The last line represents the conversion of the $\rho_{c d}(t)$ coherence into a population $\rho_{a a}(t)$ by simultaneous transfer of coherences. Similarly, the evolution of intraband coherences include non-secular coherence transfer terms similar to those of Eq. (13).

Assuming that the coherence time of the bath is much shorter than the dynamics of the electronic system we can perform the limit $t \rightarrow \infty$ in all the integrations and we obtain a time-independent relaxation tensor with constant relaxation, dephasing and coherence transfer rates. The discussion of the possible effects of coherence transfer on optical spectra of molecular systems becomes much simpler in this approximation. Here, we adopt the point of view that while for studying the real molecular systems it is desirable to perform calculations with the full time dependent rate theory, to obtain ease of interpretation it is necessary first to study the effects of the simpler constant relaxation tensor. In many applications, the so-called secular approximation is used, which neglects coherence transfer rates between transitions with different frequencies and decouples population evolution from the evolution of coherences. However, in consideration of coherence transfer effects we must avoid this approximation.

\section{Energy relaxation and optical spectra}

\subsection{Linear absorption}

The most important observation from the above theories is that the optical coherence elements of the RDM $\rho_{a 0}(t)$ do not mix with those of the one- and two-exciton bands, in other words, there are 
no elements $R_{a b c 0}$ or $R_{a 0 c d}$ of the Redfield tensor that are non-zero. Consequently, there is no direct influence of the population dynamics on absorption spectrum in the linear theory and the two blocks of the RDM can be treated separately. The dephasing terms due to relaxation exist nevertheless (see Eq. (12)). In the calculation of linear absorption spectra we need to deal with optical coherence elements of the density matrix only. Using first-order response theory the absorption coefficient $\alpha(\omega)$ can be written in the state representation as

$$
\alpha(\omega) \approx 2 \operatorname{Re} \int_{0}^{\infty} \mathrm{d} \tau \mathrm{e}^{\mathrm{i} \omega \tau} \sum_{n=1}^{N} d_{0 n} \tilde{\rho}_{n 0}(t),
$$

where $\tilde{\rho}(t)$ is the solution of the equation of motion for the density matrix elements with the initial condition $\tilde{\rho}_{n 0}(t=0)=\sum_{n=1}^{N} d_{n 0}$, with $d_{n 0}$ being the elements of the transition dipole moment operator $V=\sum_{n} d_{n 0}|n\rangle\langle 0|+$ h.c. Operator elements $\tilde{\rho}_{n 0}(t)$ and $d_{n 0}$ can be ordered into vectors $\left.|\rho\rangle\rangle=\sum_{n} \tilde{\rho}_{n 0}(t)|n\rangle\right\rangle$ and $\left.\left.|d\rangle\right\rangle=\sum_{n} d_{n 0}|n\rangle\right\rangle$, and the Liouville superoperator and the Redfield tensor elements $L_{a 0, a 0}$ and $R_{a 0, b 0}$ into matrices $\left.L=\sum_{a} \omega_{a 0}|a\rangle\right\rangle\left\langle\langle a|\right.$ and $\left.\left.R(t)=\sum_{a b} R_{a 0, b 0}(t) \mid a\right\rangle\right\rangle\langle\langle b|$, respectively. Here, we used well known Liouville space formalism (see e.g. [18]). The equation of motion for the coherences can then be expressed in a convenient matrix form

$$
\left.\left.\left.\frac{\partial}{\partial t}|\rho\rangle\right\rangle=-\mathrm{i} L|\rho\rangle\right\rangle-R(t)|\rho\rangle\right\rangle
$$

and its solution can be obtained by exponentiation of the operator $-\mathrm{i} L-R(t)$. If both $L$ and $R$ are diagonal, which is always the case for $L$ in the eigenstate representation and for $R(t)$ in secular approximation, Eq. (16) leads to as set of independent equations for the density matrix elements $\tilde{\rho}_{n 0}$ with the solutions $\tilde{\rho}_{n 0}(t)=\tilde{\rho}_{n 0}(0) \exp \left\{\mathrm{i} \omega_{n 0} t-g_{n n}(t)-\int_{0}^{t} \gamma_{n}(\tau) \mathrm{d} \tau\right\}$. A very simple interpretation of this equation can be given in ultra-short bath correlation time limit, where $g_{n n}(t) \approx g_{n} t$ and $\gamma_{n}(t) \approx \frac{1}{2} K_{n n}$. From Eq. (15) it follows that both the pure dephasing rate $g_{n}$ and the relaxation rate $K_{n n}$ contribute to the spectrum by broadening the absorption line. Note that if both rates are temperature dependent, the line width will be temperature dependent. The position of the band is however temperature independent.

If we stay in the limit of ultra-short bath correlation time, we can demonstrate that the presence of coherence transfer leads necessarily to a temperature dependent absorption band position, as observed in the photosynthetic reaction center and other photosynthetic aggregates $[15,16]$. Let us consider a simple model of two excited states $|1\rangle$ and $|2\rangle$ with energies $\epsilon_{1}$ and $\epsilon_{2}$ and coupling energy $J$. The two levels interact with a fast bath that leads to the energy transfer between the levels, dephasing of the coherences with dephasing rates $\gamma_{11}$ and $\gamma_{22}$, and coherence transfer with rates $\gamma_{12}$ and $\gamma_{21}$. The time evolution of the system will be governed by the Liouville equation with the time independent matrix

$$
M=\mathrm{i} L+R=\left(\begin{array}{cc}
\mathrm{i} \omega_{10}+\gamma_{11} & \gamma_{12} \\
\gamma_{21} & \mathrm{i} \omega_{20}+\gamma_{22}
\end{array}\right) .
$$

Here, $\omega_{n 0}=\left(\epsilon_{n}-\epsilon_{0}\right) / \hbar$, dephasing rates are defined as $\gamma_{n n}=g_{n}+\frac{1}{2} K_{n n}$, and $\gamma_{m n}$ are the coherence transfer rates from Eq. (12). The matrix (17) can be diagonalized by changing into a new "eigenstate basis". The absorption spectrum, Eq. (15) can be evaluated in this basis, too, and its absorption maxima will correspond to eigenvalues of the matrix $M$. The two eigenvalues read

$$
\bar{\omega}_{1,2}=\frac{\omega_{10}+\omega_{20}+\mathrm{i}\left(\gamma_{11}+\gamma_{22}\right)}{2} \pm \frac{1}{2} \sqrt{\left[\omega_{10}-\omega_{20}+\mathrm{i}\left(\gamma_{11}-\gamma_{22}\right)\right]^{2}-4 \gamma_{21} \gamma_{12}} .
$$


The real part of the frequency $\bar{\omega}_{1}$ gives the absorption maximum of the first band, whereas its imaginary part results in the broadening of the band. Also, even if the coherence transfer rates were temperature independent, the temperature dependence of the relaxation rates (e.g. due to detailed balance condition) makes the dephasing rates $\gamma_{11}$ and $\gamma_{22}$ temperature dependent, and as a consequence, the band maximum also shifts with temperature.

\subsection{Role of reorganization energy in coherence transfer}

It was observed previously [13], that the effect of the temperature dependent band shift depends on the reorganization energies of the two states involved in resonance interaction. This conclusion can be derived directly from the Redfield equations [14]. Starting from the same two level model, the eigenstates of the system read $|\overline{1}\rangle=\cos \theta|1\rangle+\sin \theta|2\rangle$ and $|\overline{2}\rangle=-\sin \theta|1\rangle+\cos \theta|2\rangle$, where the so-called mixing angle $\theta$ is defined as $\theta=\frac{1}{2} \arctan \left(2 J /\left(\epsilon_{1}-\epsilon_{2}\right)\right)$. The coherence transfer rates read in this case $\gamma_{12}=\Gamma_{1112}^{\infty}+\Gamma_{1222}^{\infty}$ and $\gamma_{21}=\Gamma_{2111}^{\infty}+\Gamma_{2221}^{\infty}$. We denote $\tilde{C}_{m n}(\omega)$ the Fourier transform of the bath correlation function $\tilde{C}_{m n}(\omega)=\int_{0}^{\infty} \mathrm{d} t C_{m n}(t) \mathrm{e}^{\mathrm{i} \omega t}$. For our simplified model, we assume that the correlation function at different sites differ only by the reorganization energy and that the fluctuations of the nuclear DOF on different sites are not correlated. Thus, we define $\tilde{C}_{m n}(\omega)=\delta_{m n} \lambda_{n} \bar{C}(\omega)$, where $\bar{C}(\omega)=\tilde{C}_{11}(\omega) / \lambda_{1}$. The coherence transfer rate yields

$$
\gamma_{12}=\frac{\lambda_{2}+\lambda_{1}}{\lambda_{1}} \sin \theta \cos \theta\left[\left(\sin ^{2} \theta-\frac{\lambda_{1}}{\lambda_{1}+\lambda_{2}}\right) \bar{C}\left(\omega_{12}\right)+\left(\cos ^{2} \theta-\frac{\lambda_{1}}{\lambda_{1}+\lambda_{2}}\right) \bar{C}(0)\right],
$$

and similarly for $\gamma_{21}$. The values of the parameter $\gamma_{12} \gamma_{21}$ that determines the absorption spectrum maximum for the different values of reorganization energies and mixing angles $\theta$ are plotted in Fig. 1 using overdamped Brownian oscillator model for the correlation function $C(t)$ [18]. From Eq. (19) and Fig. 1 it can be for example concluded that for a homodimer, i.e. for $\lambda_{1}=\lambda_{2}$ and $\theta=\pi / 4$ the coherence transfer rate $\gamma_{12}$ is equal to zero. Figure 1 suggests that the largest value of $\gamma_{12} \gamma_{21}$ is for large reorganization energy differences and large values of the mixing angle $\theta$. Such a situation is characteristic for the case of exciton-charge transfer state interaction $[13,14]$.

Temperature dependent absorption band shift is demonstrated in Fig. 2. The absorption spectrum broadens substantially with temperature, the shift of the absorption maximum is however clearly observable. It is necessary to note that the time-independent rate theory and the Brownian oscillator form of the correlation function that we use here to demonstrate the effect leads to unrealistically large width of the transition. To achieve more realistic line shapes it was necessary to consider non-linear form of the energy gap operator, $\Delta V(Q)$, in $[13,14]$.

\subsection{Two-dimensional coherent photon echo spectroscopy}

The time evolution of the RDM of the system can be to some extent observed by ultra-fast four-wave mixing (FWM) experiments among which two-dimensional (2D) Fourier transformed photon echo is one of the most promising recent additions [5,6]. Energy relaxation can be directly observed on the 2D spectrum which represents an excitation-stimulated emission correlation plot. The presence of electronic coherence manifests itself as beating of the diagonal and off-diagonal peaks in 2D spectrum [11].

The signal observed in FWM experiments is related to the third order response functions that can be in general represented as a sum of terms containing three time evolutions of the density matrix

$$
R\left(t_{3}, t_{2}, t_{1}\right) \approx \sum d_{0 a} d_{c 0} d_{d 0} d_{0 f} \mathcal{U}_{f 0 e 0}\left(t_{3}\right) \mathcal{U}_{e d c b}\left(t_{2}\right) \mathcal{U}_{0 b 0 a}\left(t_{1}\right)
$$




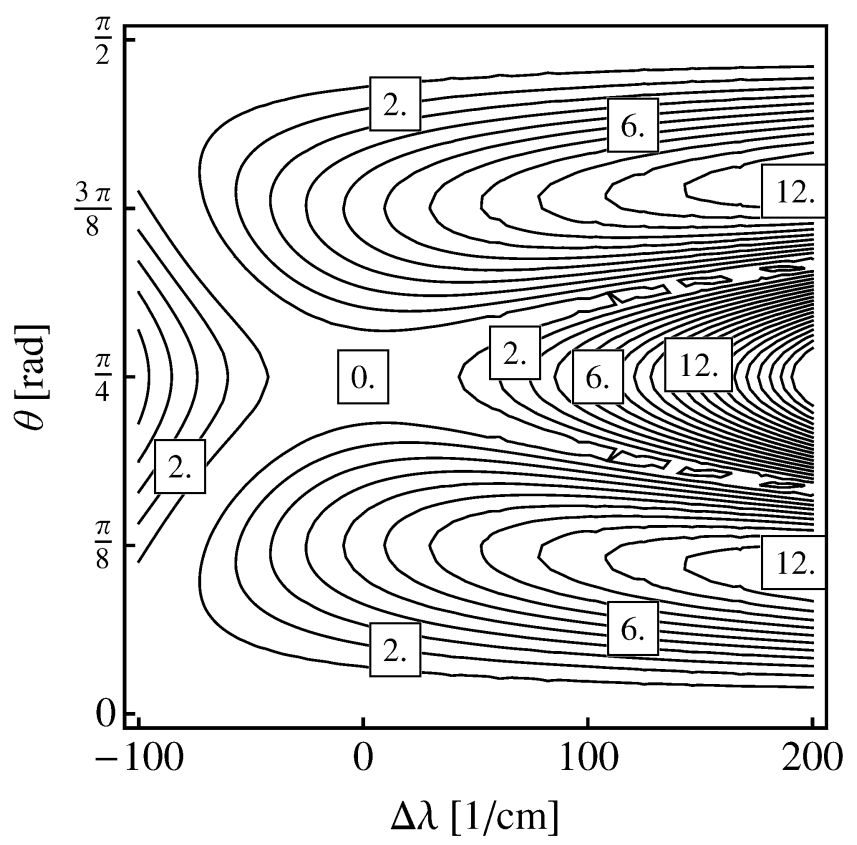

Fig. 1. Contour plot of the product of coherence transfer rates $\gamma_{12} \gamma_{21}$ as a function of mixing angle $\theta$ and the reorganization energy difference $\Delta \lambda=\lambda_{2}-\lambda_{1}$ for $\lambda_{1}=100 \mathrm{~cm}^{-1}$, constant $\Delta E=E_{2}-E_{1}=300 \mathrm{~cm}^{-1}$, correlation time $\tau_{c}=200 \mathrm{fs}$ using the high temperature overdamped Brownian oscillator form of the energy gap correlation function. Numbers give the value of the product in units of $10^{-3} \mathrm{fs}^{-2}$ for selected contours.

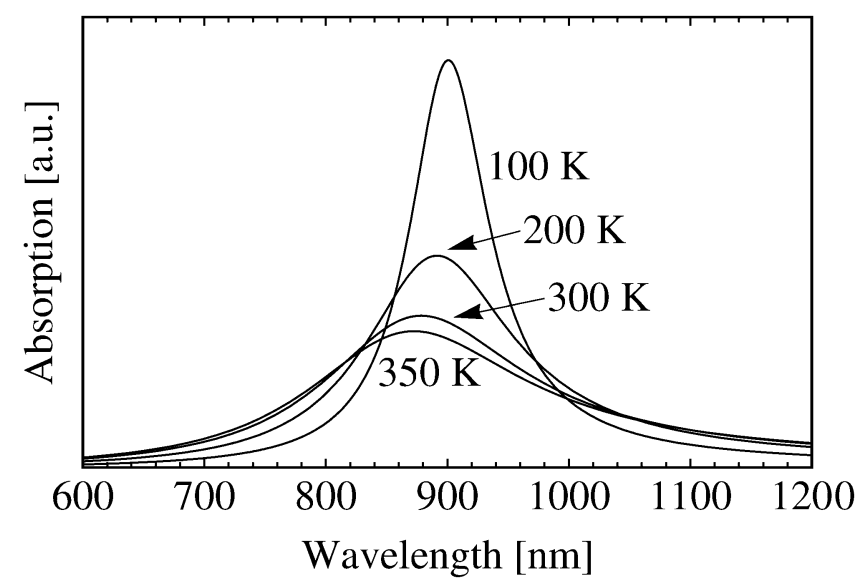

Fig. 2. Temperature dependent shift of a homogeneous Lorenzian absorption band at $T=100,200,300$ and 350 K. Dephasing and coherence transfer rates calculated using Redfield tensor with overdamped Brownian oscillator model for energy gap correlation function and parameters: $E_{1}=12,000 \mathrm{~cm}^{-1}, E_{2}=12,700 \mathrm{~cm}^{-1}$, coupling $J=1500 \mathrm{~cm}^{-1}, \lambda_{1}=100 \mathrm{~cm}^{-1}$, $\lambda_{2}=400 \mathrm{~cm}^{-1}$ and correlation time $\tau_{c}=200 \mathrm{fs}$. Only one of the two absorption lines for each temperature is plotted.

Here, the summation is performed in indices $a, b, c, d, e, f$ that run over electronic states of the system, and we used an evolution superoperator $\mathcal{U}(t)$, which gives the time evolution of the density matrix elements $\rho_{a b}(t)=\sum_{c d} \mathcal{U}_{a b c d}(t) \rho_{c d}(t=0)$. Equation (20) has to be understood as a symbolic depiction of the response theory, the rigorous derivation of the response function form can be found e.g. in $[6,18]$. In 
the limit of ultrafast laser pulses, the signal observed is directly proportional to the sum of four standard response functions (see e.g. [18]). The two-dimensional spectrum is obtained by Fourier transforming the signal in $t_{1}$ and $t_{3}$ time variables. Usually, only the real part of such a double Fourier transform is presented. The $2 \mathrm{D}$ spectrum contains two absorption spectra-like factors connected by the excited state evolution superoperator $\mathcal{U}_{e d c b}\left(t_{2}\right)$ (in general also ground state evolution operator is present). The term containing $t_{3}$ is formally similar to the one for the absorption spectrum, however, it starts with several different possible initial conditions. Consequently, it contains absorption, stimulated emission and excited state absorption contributions. The only time variable that remains is so-called population $t_{2}$. Thus, the $t_{2}$ evolution of $2 \mathrm{D}$ spectrum that is determined by the excited state evolution superoperator provides a window into the dynamics of excited states including coherences. Although the observed dynamics is distorted by the presence of transition dipoles in the response function, Eq. (20), and by the spectrum of real laser pulses, the 2D spectroscopy and photon echo spectroscopy in general provides the most sensitive method for observation of electronic coherence in coupled molecular systems $[8,12]$. Recently, 2D methods that used differently polarized laser pulses enabled control of the magnitude of different contributions to the sum, Eq. (20), resulting in higher sensitivity to desired parts of the signal [10].

\section{Manifestation of coherence transfer in photosynthetic systems}

\subsection{Temperature dependence of absorption spectra}

A puzzling temperature dependence of the lowest excitation energy band of the reaction center of Rhodobacter sphaeroides has been observed in the range of temperatures between 5 and $295 \mathrm{~K}$ [15]. Theoretical modeling of the shift is very difficult within the standard exciton model, where reorganization energies of all involved electronic levels are similar, as these states are identified with the excited states of the almost identical BChls forming reaction center's special pair (SP). As discussed above, under such conditions, coherence transfer can be neglected in the excitonic model and the relaxation dynamics leads to absorption band broadening only. Usually, to achieve the temperature dependent band shift in agreement with experimental spectrum, it was assumed that the coupling energy of the SP BChls varies with temperature (see e.g. [27]). It was however pointed out [13], that the presence of a CT state in the SP can lead to the correct band shift, provided also the non-linear contribution to the system bath coupling is considered. The non-linearity in the bath part of the system-bath Hamiltonian, Eq. (8), does not change the form of the relaxation operator, but it greatly enhances the band shift. We have formulated the theory for the same model situation [14] using the time dependent Redfield tensor. Because the energy of interaction between the $\mathrm{BChl}$ in the SP is significantly higher than the energy of their interaction with the rest of the BRC one can obtain a realistic mode of BRC by extending the two state model of [13] to include two excitonic states, and two possible CT states of the SP of BChls. Figure 3 presents the comparison of the experimental spectra of the red band of BRC at 15 and $295 \mathrm{~K}$ with such a four state model. To calculate the evolution of the coherences of the BRC including coherence transfer terms we used the time-dependent Redfield tensor from Section 2.3 and considered system bath coupling non-linear in the bath coordinates. From our calculations we concluded that the two CT states lie about 500 and $1500 \mathrm{~cm}^{-1}$ higher than the site energies of the SP BChls and their reorganization energy is of the order of $1000 \mathrm{~cm}^{-1}$ [14]. To achieve even better agreement between measurement and theory, other excited states of the BRC need to be included as well as more realistic form of the bath correlation function. In general, however, considering coherence transfer effects enables to predict temperature 


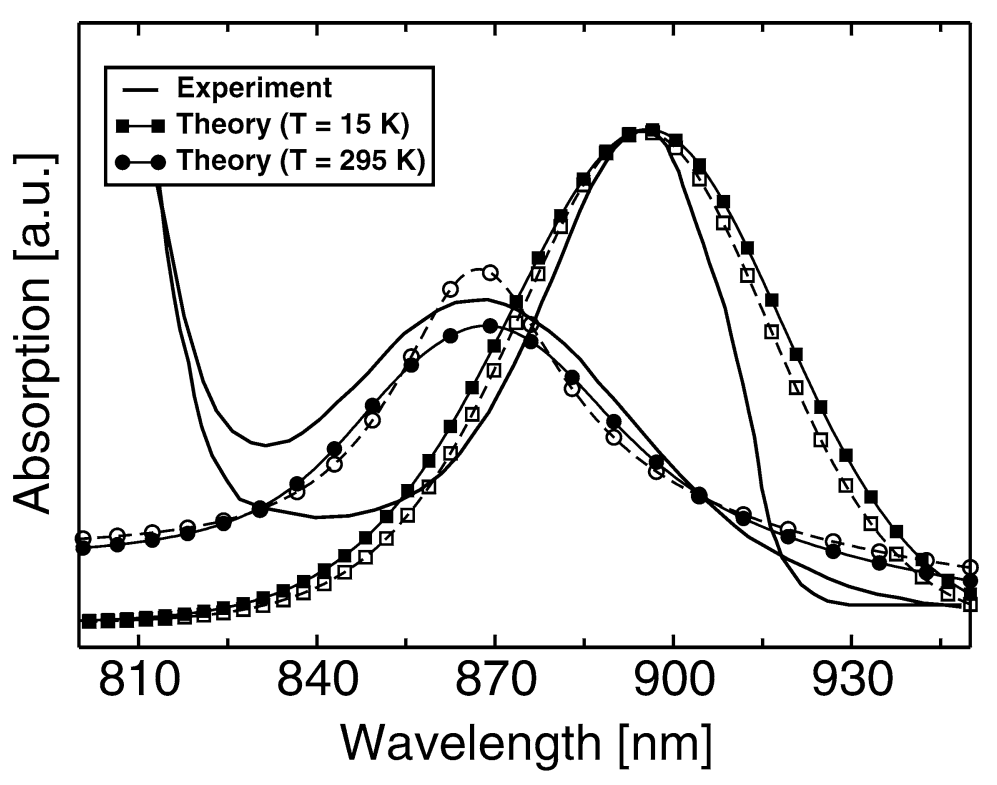

Fig. 3. Comparison of the experimental temperature dependent shift of red absorption band of the reaction center of Rhodobacter sphaeroides with calculated spectra for the same band at $T=15 \mathrm{~K}$ and $T=295 \mathrm{~K}$, parameters as in [14].

dependence of the absorption band with only a slight extension of the well-known excitonic model and thus enables application of the existing theoretical framework to a description of this rather common, but puzzling effect.

\subsection{Photon echo spectra}

In this section, we present the main results of a heterodyne detected 2D Fourier transformed photon echo experiment on FMO complex of photosynthetic bacteria Chlorobium tepidum [8]. This experiment was aimed at observing oscillations caused by the evolution of electronic coherence during the so-called population time $t_{2}$ (see Eq. (20)). The coherence, a result of coherent superposition of electronic states, in many ways analogous to vibrational wavepackets, is formed in the system by excitation by a short laser pulse. Theoretical predictions [11] indicate that both the amplitudes and shapes of the 2D spectral features contain beating patters with frequencies corresponding to energy differences between excitonic states involved in the superposition.

Two-dimensional spectra were taken at 33 population times $t_{2}$, ranging from 0 to 660 fs. Representative spectra are show in Fig. 4. Here, the lowest energy excitonic level gives rise to a diagonal peak near $825 \mathrm{~nm}$. This peak clearly oscillates: its amplitude grows, fades and subsequently grows again. The peak's shape evolves with these oscillations, becoming more elongated when weaker and more rounded when the signal amplitude intensifies. The associated cross peak amplitude also appears to oscillate, although its increasing amplitude results from relaxation of the population of higher lying excitonic states. Surprisingly, the quantum beating lasts at least for $600 \mathrm{fs}$. Both these observations contrast with the general assumption that the coherences responsible for such oscillations are destroyed very rapidly, and that population relaxation proceeds with complete destruction of coherence. The appearance of the relaxation cross peak oscillations strongly indicates that the energy relaxation in FMO is accompanied by coherence transfer, in other words, the superposition form of the excitation does not get destroyed during relaxation on a short time scale. 

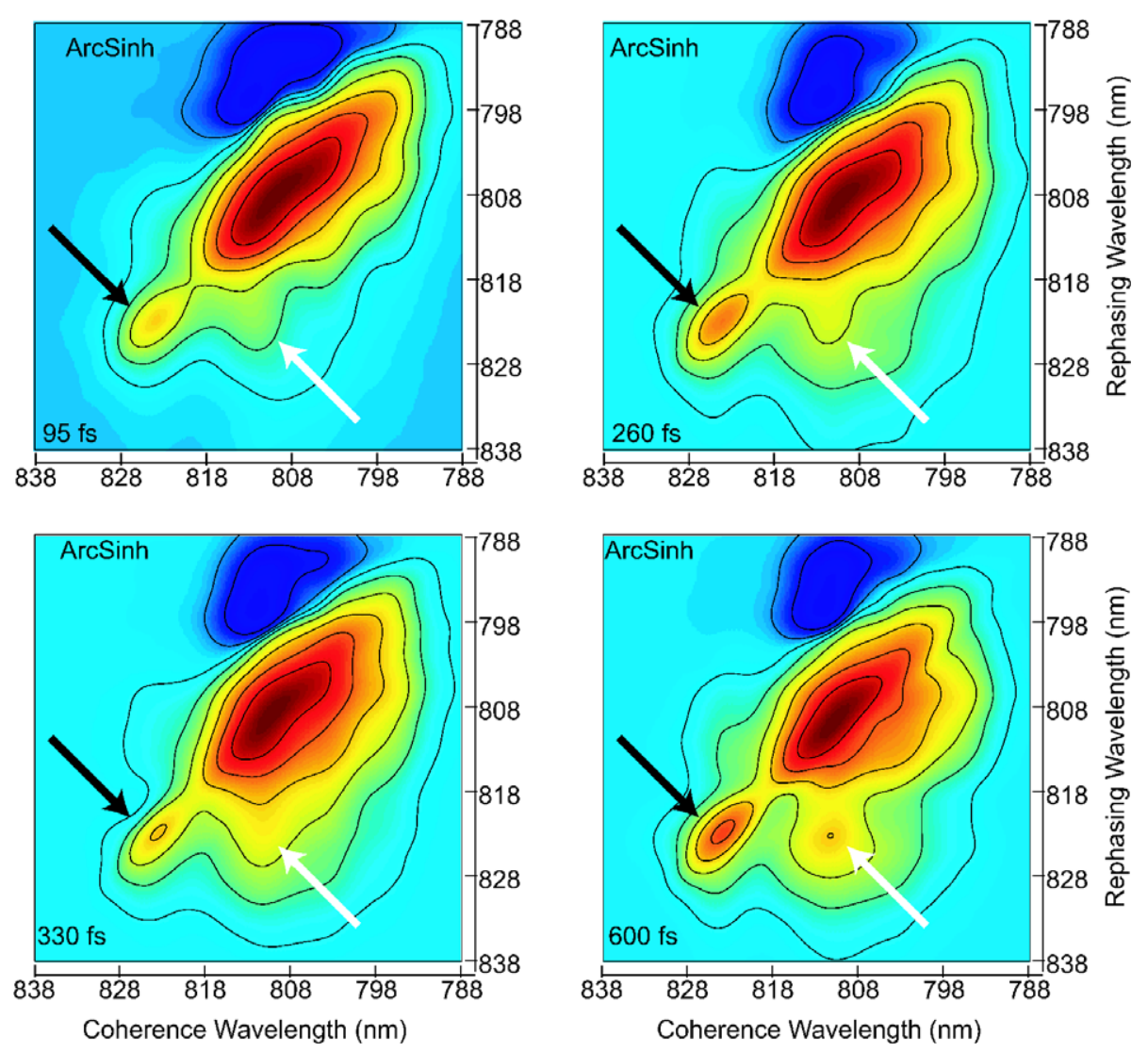

Fig. 4. Experimental 2D spectra of the Chlorobium tepidum FMO complex at $77 \mathrm{~K}$ at population times $t_{2}=95,260,330$ and $600 \mathrm{fs}$. The figure demonstrates the emergence and oscillation of the exciton peak, due to relaxation and coherence transfer (white arrow) and oscillation of the diagonal peak (black arrow). The data are shown with the arcsinh (logarithmic) scale to enhance smaller features.

The observations of [8] demonstrate the necessity to fully include description of coherence into a theoretical treatment of energy relaxation in photosynthetic complexes. While the dephasing will destroy the ensemble coherence much faster at physiological conditions, quantum coherence will still be important in relaxation dynamics of individual molecules, because their superposition states do not depend on dephasing of the ensemble. The relaxation proceeding in, at least partially, coherent way might lead to higher efficiency of energy transfer in photosynthetic systems. The superposition state formed by an initial fast excitation might be able to reversibly sample relaxation rates from all component excitonic states thus using all advantages of the quantum superposition. Such an energy relaxation mechanism may be comparable to a mechanism underlying quantum computation [8]. Two-dimensional spectroscopy provides a unique experimental window into this new realm of biophysics and might provide answers to questions about the role of coherent evolution in photosynthetic and other biological systems.

\section{Conclusions}

Recent experimental achievements in realization of coherence sensitive two-dimensional photon echo spectroscopy and observation of coherent electronic wavepackets in photosynthetic complexes has in- 
spired a renewed theoretical interest in the effects of electronic coherence in molecular systems. Based on recent measurements it was proposed that electronic coherence transfer plays a significant role in relaxation of optically excited states of the Fenna-Matthews-Olson photosynthetic protein complex. This implies a breakdown of the standard secular approximation in the description of relaxation. In this contribution we demonstrate the effects of the coherence transfer on absorption spectra of another photosynthetic complex, the bacterial photosynthetic reaction center. Here, certain temperature dependencies of the line shapes can be attributed to coherence transfer between two optical transitions. Considering the two states responsible for the lowest energy band of the reaction center, we derive analytical results that demonstrate the temperature dependent shift of the band maximum and link the magnitude of the effect to the difference of the reorganization energies of the two states. Due to the large reorganization energy difference needed to explain the experimentally observed shift, the most likely explanation is that one of the involved states is a charge transfer state, as previously proposed in the literature.

\section{Acknowledgments}

This work has been supported by a grant from National Science Foundation (USA). T.M. acknowledges the kind support by the Czech Science Foundation through grant GACR 202/07/P278 and by the Ministry of Education, Youth and Sports of the Czech Republic through the research plan MSM0021620835. L.V. was supported by the Lithuanian State Science and Studies Foundation.

\section{References}

[1] R.E. Blankenship, Molecular Mechanisms of Photosynthesis, Blackwell Science, Oxford, 2002.

[2] H. van Amerongen, L. Valkunas and R. van Grondelle, Photosynthetic Excitons, World Scientific, Singapore, 2000.

[3] D.M. Jonas, Annu. Rev. Phys. Chem. 54 (2003), 425.

[4] M.L. Cowan, J.P. Ogilvie and R.J.D. Miller, Chem. Phys. Lett. 386 (2004), 184.

[5] T. Brixner, I.V. Stiopkin and G.R. Fleming, Opt. Lett. 29 (2004), 884.

[6] T. Brixner, T. Mančal, I.V. Stiopkin and G.R. Fleming, J. Chem. Phys. 121 (2004), 4221.

[7] T. Brixner, J. Stenger, H.M. Wasvani, M. Cho, R.E. Blankenship and G.R. Fleming, Nature 434 (2005), 625.

[8] G.S. Engel, T.R. Calhoun, E.L. Read, T.-K. Ahn, T. Mančal, Y.-C. Cheng, R.E. Blankenship and G.R. Fleming, Nature 446 (2007), 782.

[9] D. Zigmantas, E.L. Read, T. Mančal, T. Brixner, A.T. Gardiner, R.J. Cogdell and G.R. Fleming, Proc. Natl. Accad. Sci. USA 103 (2006), 12672.

[10] E.L. Read, T. Mančal, I.V. Stiopkin and G.R. Fleming, Proc. Natl. Acad. Sci. USA 121 (2007), 4221.

[11] A.V. Pisliakov, T. Mančal and G.R. Fleming, J. Chem. Phys. 124 (2006), 234505.

[12] H. Lee, Y.-C. Cheng and G.R. Fleming, Science 316 (2007), 1462.

[13] T. Renger, Phys. Rev. Lett. 93 (2004), 188101.

[14] T. Mančal, L. Valkunas and G.R. Fleming, Chem. Phys. Lett. 432 (2006), 301.

[15] H. Huber, M. Meyer, H. Scheer, W. Zinth and J. Wachtveitl, Photosynth. Res. 55 (1998), 153.

[16] V. Urboniene, O. Vrublevskaja, G. Trinkunas, A. Gall, B. Rober and L. Valkunas, Biophys. J. 93 (2007), 2188.

[17] S. Vaitekonis, G. Trinkunas and L. Valkunas, Photosynth. Res. 86 (2005), 185.

[18] S. Mukamel, Principles of Nonlinear Spectroscopy, Oxford University Press, Oxford, 1995.

[19] S. Jang, M.D. Newton and R.J. Silbey, Phys. Rev. Lett. 92 (2004), 218301.

[20] G.D. Scholes, X.J. Jordanides and G.R. Fleming, J. Phys. Chem. B 105 (2001), 1640.

[21] V. May and O. Kühn, Charge and Energy Transfer Dynamics in Molecular Systems, Wiley-VCH, Berlin, 2000.

[22] H.-P. Breuer and F. Petruccione, The Theory of Open Quantum Systems, Clarendon Press, Oxford, 2002.

[23] H.-P. Breuer, B. Kappler and F. Petruccione, Phys. Rev. A 59 (1999), 1633.

[24] W.M. Zhang, T. Meier, V. Chernyak and S. Mukamel, J. Chem. Phys. 108 (1998), 7763.

[25] M. Yang and G.R. Fleming, Chem. Phys. 275 (2002), 355.

[26] G. Lindblad, Commun. Math. Phys. 48 (1976), 119.

[27] X.J. Jordanides, G.D. Scholes and G.R. Fleming, J. Phys. Chem. B 105 (2001), 1652. 


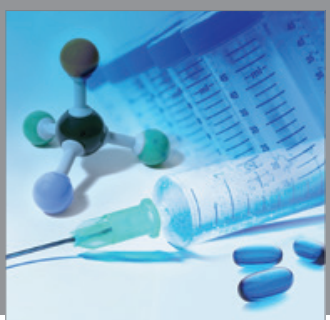

International Journal of

Medicinal Chemistry

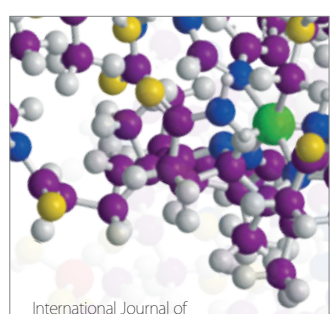

Carbohydrate Chemistry

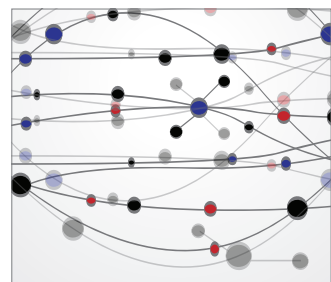

The Scientific World Journal
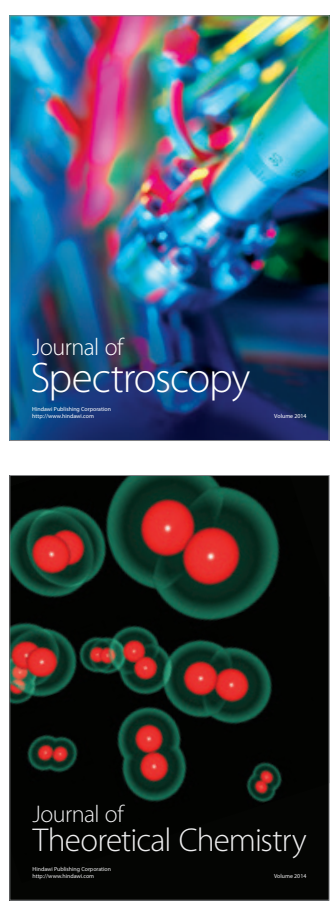
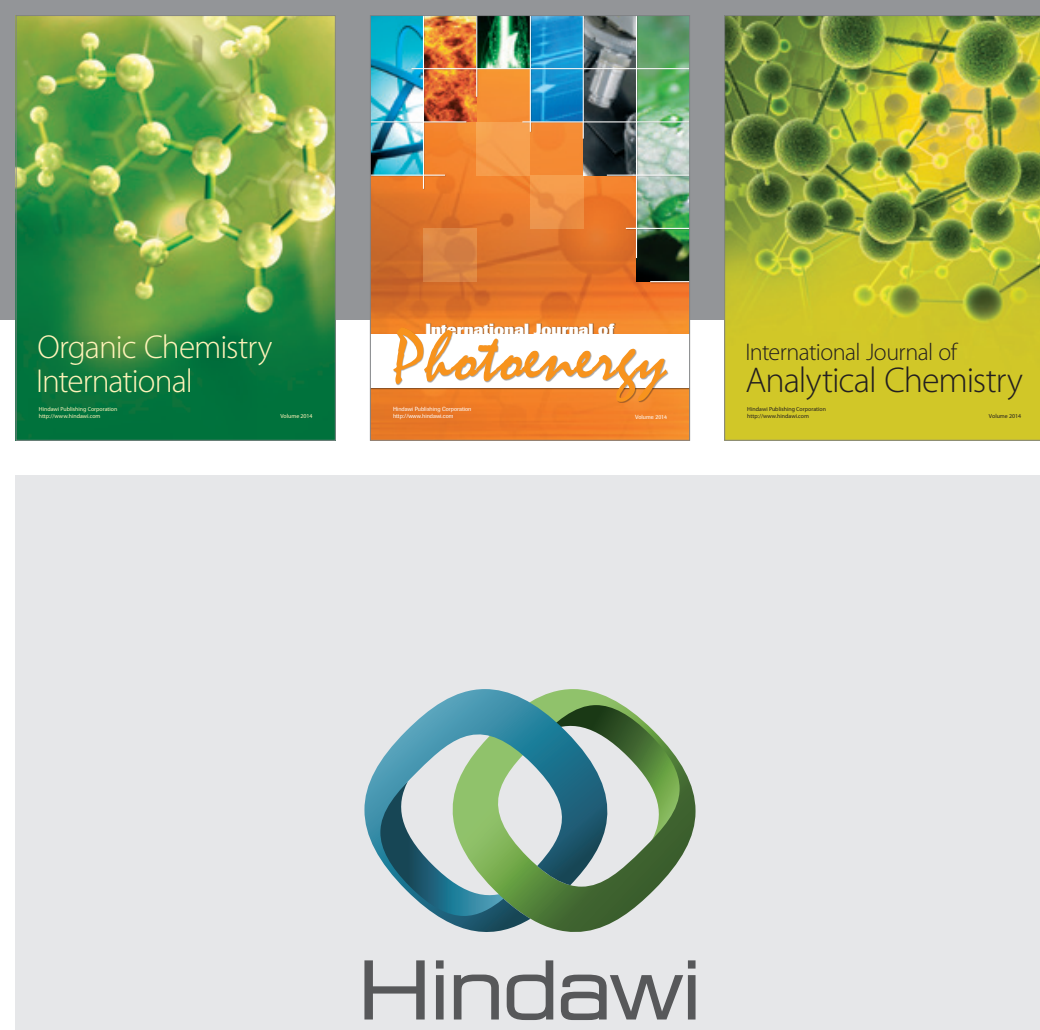

Submit your manuscripts at

http://www.hindawi.com
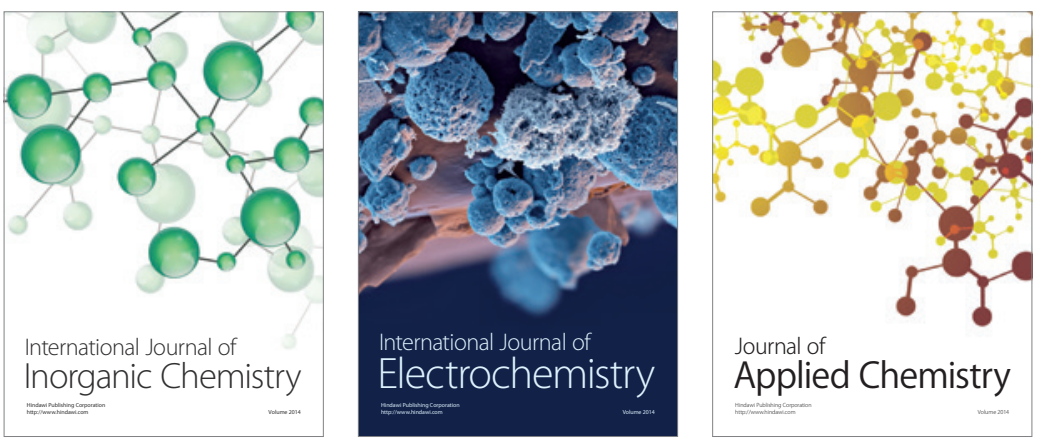

Journal of

Applied Chemistry
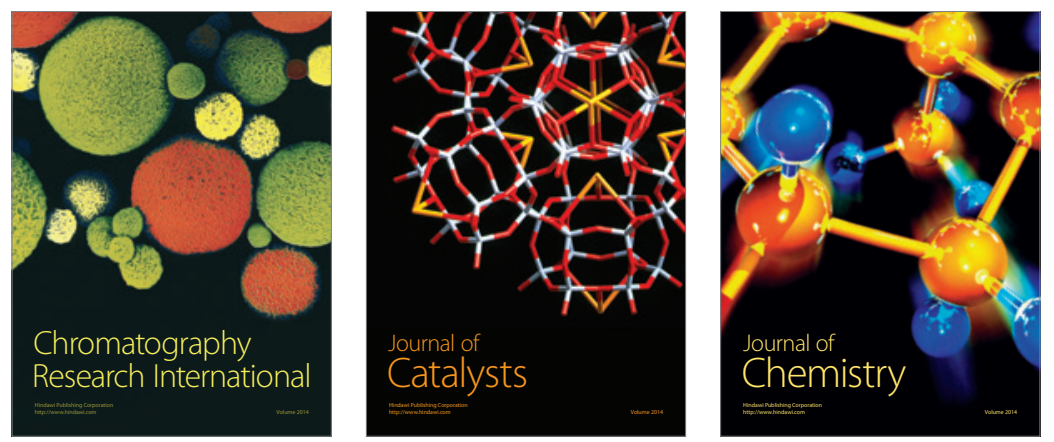
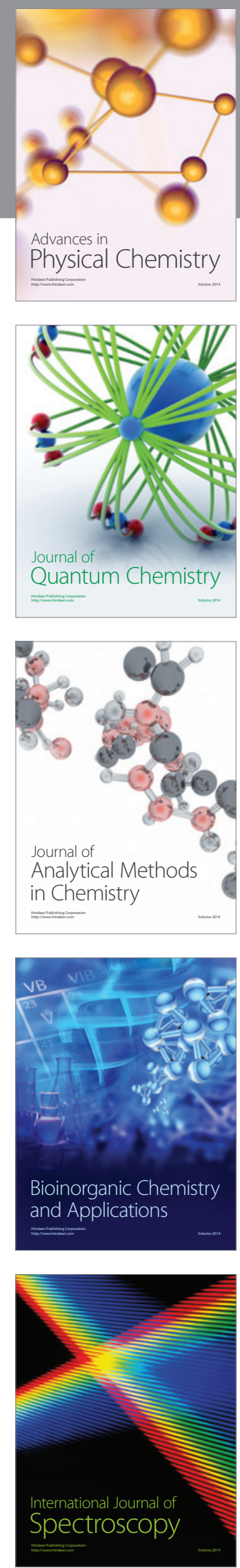